\title{
On Enhancing a Hybrid Admission Control Algorithm for IEEE 802.11e EDCA
}

\author{
Mohamad El Masri, Guy Juanole and Slim Abdellatif
}

\author{
Mohamad EL MASRI · Guy Juanole · Slim Abdellatif \\ LAAS-CNRS ; Université de Toulouse ; 7, avenue du Colonel Roche, F-31077 Toulouse, France \\ e-mail: \{masri, juanole, slim\}@laas.fr
}

\begin{abstract}
Call Admission Control is one of the main mechanisms allowing an efficient protection of active flows, especially on a wireless network using a distributed access scheme as is the case for IEEE 802.11e EDCA. We proposed in a previous paper [5] a hybrid admission control algorithm (based on analytical model and on measurements) for EDCA which have proven to perform well when compared to another reference algorithm. We present here general modifications to hybrid admission control algorithms. These modifications are applied to our proposed algorithm and analyzed via simulation. Analysis clearly shows the advantages of these modifications.
\end{abstract}

\section{Introduction}

Providing quality of service (QoS) within an access network is one of the main challenges facing wireless access networks nowadays. Recent advances in this area include the standardization in 2005 of the QoS amendments to the IEEE 802.11 standard [1] (done by the IEEE 802.11e working group [2]). 802.11e introduced a new access function called the Hybrid Coordination Function (HCF) including one distributed access mechanism (the Enhanced Distributed Channel Access - EDCA) and a centralized polling based mechanism (the HCF Centralized Channel Access - HCCA). EDCA is a CSMA/CA based distributed channel access with traffic differentiation. EDCA classifies packets into 4 access categories with a different access probability each. The 4 access categories (AC) defined by EDCA are: Voice (AC_VO), Video (AC_VI), Best Effort (AC_BE) and Background (AC_BK).

One of the main mechanisms used in order to protect the time sensitive flows using the network from incoming flows is admission control. A flow wishing to use a network in which admission control is applied must first request admission to the network; the admission control's mission is to protect already admitted flows from incoming ones: it thus must decide whether to accept the requesting flow or not based on the incoming flow's specification and on the network's state. The 802.11e working group specifies a framework for admission control algorithms including main procedures and the necessary signaling messages but leaves the specification 
of the algorithm per se to manufacturers when deemed relevant.

Several admission control algorithms have been proposed for IEEE 802.11e in literature. Gao et. al. [6] give an overview of some of them. Gao et. al. divide the EDCA admission control algorithms into measurement based algorithms (those basing the admission decision on measurements of network status) and model based (those using an analytical model to assess the behavior of the network).

In [5] we proposed a hybrid admission control algorithm (based both on an analytical model and on measurement of network specific metrics). The algorithm we proposed is made out of three interacting blocks: a parameterized Markov chain model of a saturated Access Category allowing the calculation of a maximum achievable throughput for a given flow; an estimation process of future collision rates and busy probabilities based on actual measurements made on the medium, those estimations are injected to the model in order to make the achievable throughput calculation more accurate; and an admission decision process based on information on the specifications of the flow requiring admission and on the calculated achievable throughput. Extensive simulations were made and the algorithm we proposed proved to perform better than a reference model based algorithm [7]. However, in some cases, the algorithm admitted flows in excess. We propose in this paper enhancements to correct this flaw that can be applied to any similar hybrid algorithm.

The modifications we present here are introduced to both the estimation process and to the decision process. Two main ideas steer those modifications: one is to condition the estimation process and the decision process by the medium state leading to more drastic refusals, the other is to introduce feedback correction to the estimation process of busy probabilities.

The paper presents as follows: the next section details the admission control algorithm we presented in [5] including a brief introduction to the Markov chain model used within the algorithm. The following section presents the enhancements we propose to the algorithm. The fourth section presents, by means of simulation, the analysis and the comparison of the enhancements. Conclusion and future work are given at the end of the paper.

\section{The hybrid admission control for EDCA}

In this section we present the hybrid admission control algorithm for EDCA. The algorithm was thoroughly presented in [5]. We first briefly present the Markov chain model upon which is based the admission control algorithm for EDCA. We then present the different aspects of the algorithm. 


\subsection{Introducing the model}

We designed a discrete Markov chain model of an EDCA Access Category (AC) behavior. The model included all the standard access functions: differentiated deference and backoff procedure, different collision situations, virtual collision, retransmissions and drops among other mechanisms. This general model was presented in [3]. The general model was later reduced into a three-useful-state model and presented in [4]. From this reduced model several closed form performance metrics were derived among which is the achievable throughput of a saturated Access Category Queue in a specified environment (represented by the collision probability $p_{i}$ and the probability of the medium becoming busy $p_{b}$ ). The synthetic model is shown in figure 1. The synthetic model is composed of three useful states: the Access Attempt state labeled 1, the successful transmission state labeled 2 and the drop state labeled 3. The Access Attempt state represents the state of an $A C$ (which we call $A C_{i}$ ) trying to transmit a packet (going through several unsuccessful transmission attempts, the backoff times preceding those attempts and the backoff time preceding the supposedly successful transmission attempt). The successful transmission state and the drop state represent respectively the fact that a packet's transmission was possible or not (the retransmission threshold being reached). The transitions between the states are labeled with the transition probability and the transition time (transition time from state 3 to state 1 is null, we suppose that after a packet drop, the access category proceeds instantaneously with the access attempt of a new packet). $T_{T}$ is the time it takes $A C_{i}$ to transit from the Access Attempt state to the Successful transmission state (i.e. the access delay of a successfully transmitted packet), this occurs with probability $P_{T} . T_{D}$ is the time it takes $A C_{i}$ to transit from the Access Attempt state to the Drop state with probability $P_{D}$ (i.e. the packet's retransmission threshold -noted $m+h$ - was reached, the packet is dropped).

This model is a discrete Markov chain. We can evaluate by means of the transition probabilities and the transition times the equilibrium probabilities of states 1,2 and 3: $\Pi_{1}, \Pi_{2}$ and $\Pi_{3}$. We get $\Pi_{1}=\frac{1}{2} ; \Pi_{2}=\Pi_{1} P_{T} ; \Pi_{3}=\Pi_{1} P_{D}$. From these probabilities we can conclude the formula of the achievable throughput of a saturated $A C$ :

\section{Throughput $_{i}=$}

Fig. 1 Abstract model of an EDCA AC behavior

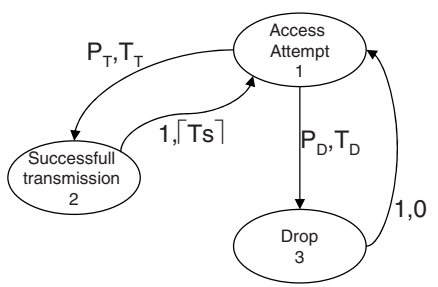


Payload being the number of slots necessary to transmit the payload.

We do not present the detailed formulas of transition times and transition probabilities of the synthetic model because of lack of space and since it is out of the scope of this paper. Note however that they are function of $p_{i}$ and $p_{b}$ among other parameters. We highly recommend the reader to check the detailed formulas in our previous work $[4,5]$.

\subsection{The algorithm}

We give a quick overview of the functioning of the algorithm and the different mechanisms used in the decision making process.

The algorithm is called when a new flow request arrives at the Access Point's admission controller. Two conditions must apply in order for the algorithm to accept the arriving flow: first the arriving flow must be able to achieve its request in terms of throughput, the second condition being that the admission of the new flow must not degrade the quality of service of already admitted flows. The algorithm bases its admission decision on two parameters:

- estimations made on what each flow's collision rate and the medium busy rate would be if the newly arriving flow was to be admitted (those estimations are made based on actual measurements as explained in next section).

- the maximum achievable throughput of each flow, in the previously estimated collision and medium business conditions, calculated using the Markov chain model of an access category presented earlier.

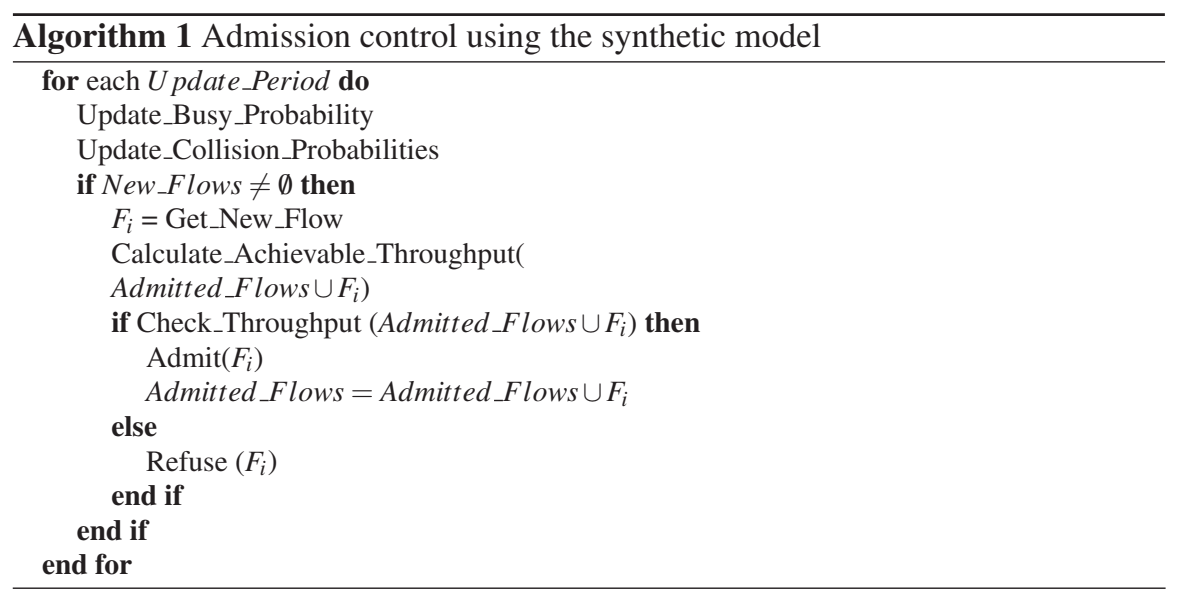

We define $F_{i}$ as the flow requesting admission, New_Flows is the set of all newly arriving flows, Admitted_Flows is the set of active flows. U pdate_Busy_Probability 
and Update_Collision_Probabilities are the procedures giving the admission controller the information he needs on both probabilities (by direct measurement for the busy probability and by piggybacking from the different flows for their collision probabilities). The procedure Calculate_Achievable_Throughput(SetofFlows) calculates for each flow (all the active flows and one newly arriving flow) their maximum achievable throughput in the estimated network conditions (i.e. for a given flow, its throughput if saturated, given the estimated busy probability and the estimated collision probability). Procedure Check_Throughput(SetofFlows) returns true if, for each of the flows in the set, its achievable throughput is greater that its request: Calculated_Achievable_Throughput $(F)>$ Requested_Bandwidth $(F)$. The algorithm is detailed in algorithm 1.

\subsection{Estimating the probabilities}

In the process of decision making, the values of busy probability $p_{b}$ and each $A C$ 's collision probability are needed. The busy probability can be directly measured by the Access Point. The collision probabilities are calculated by the stations and communicated periodically to the access point by means of piggybacking or management packets (in fact the station will communicate, for each $A C$, a count of access attempts and of collisions). We figured that since the measurements are made in the actual context of the medium (i.e. having only the already admitted flows active and not those requesting admission), the achievable throughput calculation wouldn't be correct. Thus, an additional process of estimation was added which, based on the actual measurements made and on the specification of the flow requesting to access the medium, will estimate the values of collision probability and busy probability would the requesting flow be admitted.

Let $F_{i}$ be the flow whose admission is being examined, $F_{i}$ will be using access category $A C_{i}$ in station $s$. We also define $\tau_{i}$ as the probability for $A C_{i}$ to access the medium on a free slot. We define $\Gamma_{s}$, the probability for station $s$ to access the medium. Among the access categories of a station, only one can access the medium at a specific time slot (the others are either inactive or in backoff procedure or have lost a virtual collision); we can therefore write $\Gamma_{s}=\sum_{i=0}^{3} \tau_{i}$.

We define $p_{b}$ as the probability of the medium becoming busy. We neglect the reasons of business of the medium other than station access, we therefore write

$$
p_{b}=1-\left(1-\Gamma_{1}\right)\left(1-\Gamma_{2}\right) \ldots\left(1-\Gamma_{M}\right)=1-\prod_{j=1}^{M}\left(1-\Gamma_{j}\right)
$$

$\mathrm{M}$ being the number of stations in the medium.

$p_{i r}$ is the probability for $A C_{i}$ to suffer a real collision when accessing the medium, we can write $p_{i r}$ as follows: 


$$
p_{i r}=\tau_{i}\left(1-\left(1-\Gamma_{1}\right) \ldots\left(1-\Gamma_{s-1}\right)\left(1-\Gamma_{s+1}\right) \ldots\left(1-\Gamma_{M}\right)\right)=\tau_{i}\left(1-\prod_{j \neq s}\left(1-\Gamma_{j}\right)\right)
$$

In order to better understand the following, note that all values indexed old are measured values (either directly by the access point, or measured by the stations and communicated to the access point). The values indexed new are estimated values (estimation of what would the value be if the requesting flow was active).

In the case of the collision probability, we estimate the effect of introducing $F_{i}$ on real collisions occurring on the medium. Since we consider the admission of one flow at a time, we suppose that the access activity of $F_{i}$ 's station would be the only one to change. Let $p_{\text {ir new }}$ and $\tau_{i \text { new }}$ be the estimated real collision probability of $A C_{i}$ and its estimated access probability if $F_{i}$ was to be accepted. $p_{\text {ir_old }}$ and $\tau_{i_{-} o l d}$ the actual real collision and access probabilities. We have:

$$
\begin{aligned}
& p_{\text {ir_new }}-p_{\text {ir_old }}=\left(\tau_{\text {i_new }}-\tau_{i_{-} \text {old }}\right)\left(1-\prod_{j \neq s}\left(1-\Gamma_{j \_o l d}\right)\right) \\
& p_{\text {ir_new }}=\left(\tau_{i \_n e w}-\tau_{i_{-} \text {old }}\right)\left(1-\prod_{j \neq s}\left(1-\Gamma_{j \_o l d}\right)\right)+p_{\text {ir_old }}
\end{aligned}
$$

Let $\Delta_{\tau}$ be the difference introduced by $F_{i}$ to the access category's access probability should $F_{i}$ be accepted. We have:

$$
p_{\text {ir_new }}=\left(\Delta_{\tau}\right)\left(1-\prod_{j \neq s}\left(1-\Gamma_{j \_o l d}\right)\right)+p_{\text {ir_old }}
$$

This estimated ratio will be considered as the estimation of what $A C_{i}$ 's real collision probability would be if $F_{i}$ was to be admitted. In the equation above, the access activities of the stations are communicated to the $\mathrm{HC}$ along with the information on the different active flows.

In the same fashion as above, we define $p_{b \_n e w}$ as the estimated busy probability if $F_{i}$ was to be accepted and $p_{b_{-} \text {old }}$ the actual busy probability. Since we consider the admission of one flow at a time, we suppose that the access activity of $A C_{i}$ would be the only one to change. Hence:

$$
\begin{aligned}
\frac{1-p_{b \_n e w}}{1-p_{b \_o l d}} & =\frac{\left(1-\Gamma_{1 \_o l d}\right)\left(1-\Gamma_{2 \_o l d}\right) \ldots\left(1-\Gamma_{i \_n e w}\right) \ldots\left(1-\Gamma_{M \_o l d}\right)}{\left(1-\Gamma_{1 \_o l d}\right)\left(1-\Gamma_{2 \_o l d}\right) \ldots\left(1-\Gamma_{i_{\_} o l d}\right) \ldots\left(1-\Gamma_{M_{-} \text {old }}\right)} \\
& =\frac{\left(1-\Gamma_{i \_n e w}\right)}{\left(1-\Gamma_{i \_o l d}\right)}
\end{aligned}
$$

Following the same reasoning as for the estimation of the real probability we have: 


$$
\begin{aligned}
& \frac{1-p_{b \_n e w}}{1-p_{b \_o l d}}=\frac{\left(1-\Gamma_{i \_n e w}\right)}{\left(1-\Gamma_{i \_o l d}\right)}=\frac{\left(1-\Gamma_{i_{\_} \text {old }}-\Delta_{\tau}\right)}{\left(1-\Gamma_{i \_o l d}\right)} \\
& 1-p_{b \_n e w}=\left(1-p_{b \_o l d}\right) \frac{\left(1-\Gamma_{i_{\_} o l d}-\Delta_{\tau}\right)}{\left(1-\Gamma_{i \_o l d}\right)} \\
& p_{\text {b_new }}=1-\left(1-p_{b_{-} \text {old }}\right)\left(1-\frac{\Delta_{\tau}}{\left(1-\Gamma_{i_{-} \text {old }}\right)}\right)
\end{aligned}
$$

The only unknown in both estimations is $\Delta_{\tau} . \Delta_{\tau}$ represents the additional accesses introduced by the new flow which can be additional transmission and possible retransmissions introduced by the flow. Considering only one possible collision per transmitted packet, we use the following to estimate $\Delta_{\tau}: \Delta_{\tau}=\left(1+p_{i r}\right) \delta, \delta$ being the number of accesses introduced by the flow (i.e. the number of packets to be sent during the update period). Both those estimations will be used in the calculation of the achievable throughput during the admission making process.

\section{Enhancing the algorithm}

Simulations have been made showing that the estimations we make of collision probabilities and of busy probability, although going in the correct direction, are not exact. This is mainly due to the following fact: we consider in our estimations that the new flow will affect the collision rate of its access queue alone; however, it is a fact that all other access queues will be affected by the new flow. The admission control algorithm worked correctly but did, in some cases, wrong admission decisions. Two main ideas drove the enhancements we propose:

- adding information about medium state either to the estimation process or to the decision process.

- correcting the estimations made on the different probabilities with the help of a feedback correction system.

\subsection{Additional medium information}

\subsection{1 added to the estimation process}

When estimating what busy probability $p_{b}$ or collision probability $p_{i}$ would be if flow $F_{i}$ was to be accepted, we consider that the new flow will introduce a number of accesses and collisions in correlation with the number of packets it has to send. However, it is clear that the number of collision will be greater with a greater number of flows accessing the medium or with a greater busy rate of the medium. We thus decided to introduce pessimism into the estimation process which will be in correlation with the occupation of the medium: the greater the occupation of the medium, 
the greater will be the estimated busy probability. This is done as follows: the estimated value of $p_{b}$ is multiplied by $1<\alpha<1.2$ which we will call the pessimism factor. We have $\alpha=1+0.2 *\left(1-\frac{\text { totalBandwidth-totalRequests }}{\text { totalBandwidth }}\right)$. $\alpha$ will be greater with a greater occupation of the medium.

\subsection{2 added to the decision process}

The change introduced at this level is driven by the intuition that a bad admission decision that could have been avoided is taken when the medium has a high occupation rate. The algorithm should thus be more reluctant to admit with a higher occupation rate of the medium. Our proposal to replace the usual comparison on which is based the decision by the following comparison:

$\alpha$ Calculated_Achievable_Throughput $(F)>$ Requested_Bandwidth $(F)$ where $0 \leq$ $\alpha \leq 1$ and can be written: $\alpha=(1-\beta)+\beta * \frac{\text { totalBandwidth-totalRequests }}{\text { totalBandwidth }} \beta$ will specify the degree of pessimism and was set to 0.2 for the simulations. The greater the requests, the greater $\alpha$, more pessimistic become the decisions. With $\beta$ as little as 0.2 , the decision will mainly be based on the information given by the model.

\subsection{Estimation correction}

Since the estimation process we introduced fails in giving correct results in high medium occupation periods, and as said earlier, bad decisions are usually taken in high medium occupation periods, we introduce a simple history-less feedback correction of busy probability estimation where we add to each estimation the error made on the previous one. Let $p_{b e \_}$the $k^{\text {th }}$ estimated value of $p_{b}$ (using the original estimation process), $p_{b m \_}$the $k^{\text {th }}$ measured value of $p_{b}$ and let $p_{b \_n e w}$ be the new corrected estimation of $p_{b}$. The estimation works as follows: $p_{b \_n e \downarrow}=p_{b e \_}+\left(p_{b m \_-1}-p_{b e \_-1}\right)$.

\section{Analyzing the enhancements}

We present in this section analysis we made of the three enhancements we propose to the admission control algorithm. The analysis is made by means of simulation using the network simulator (ns-2) [8]. We use the EDCA module contributed by the Telecommunication Networks Group of the Technical university of Berlin [9]. The EDCA module was modified in order to integrate the admission control we propose along with the enhancements. For each scenario we present, 10 simulations with different random number generator seeds were executed, the results we present in this section are sample means. In each simulation, a number of flows will be periodically activated, seeking thus admission to access the network through the 


\begin{tabular}{|l|c|c|c|}
\hline Scenario & Packet Size (Bytes) & Interarrival (s) & Bandwidth (Mbps) \\
\hline Scenario 1 & 600 & 0.002 & 2.4 \\
\hline Scenario 2 & 800 & 0.004 & 1.6 \\
\hline Scenario 3 & 600 & 0.004 & 1.2 \\
\hline
\end{tabular}

Table 1 Specifying the presented scenarios

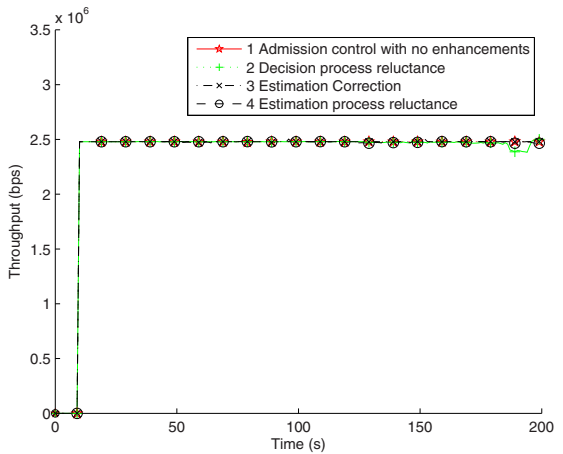

Fig. 2 Mean throughput of flows, scenario 1

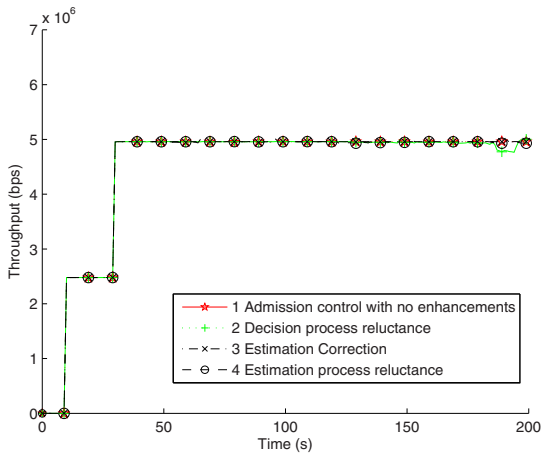

Fig. 3 Total throughput of flows, scenario 1

admission control algorithm (or through the enhanced admission control algorithm). The metrics used for the analysis are the following:

- The total throughput of all the flows in a specific scenario using the algorithm with or without the enhancements.

- The mean throughput of a flow in a specific scenario using the algorithm with or without the enhancements.

- The cumulative distribution function of the delays of all data packets.

Different execution scenarios were tested, we present in the following the results of several representative scenarios. In scenarios 1, 2, and 3: the channel is considered error free, no hidden terminals are present and the stations function at 11 Mbps. One station operates as the Access Point and will execute the admission control algorithm. Within the other stations CBR flows with the traffic specifications described in table 1 will be periodically activated, thus requesting access to the admission controller. The results of those simulations are presented in figures 2-10.

\subsection{Analysis}

Scenarios 1, 2 and 3 presented here are representative of the different behaviors encountered for different simulation scenarios tested. Note that the bad admission decisions of the original admission control algorithm are not generalized. The algorithm works well but does in some cases bad admission decisions, hence the intro- 

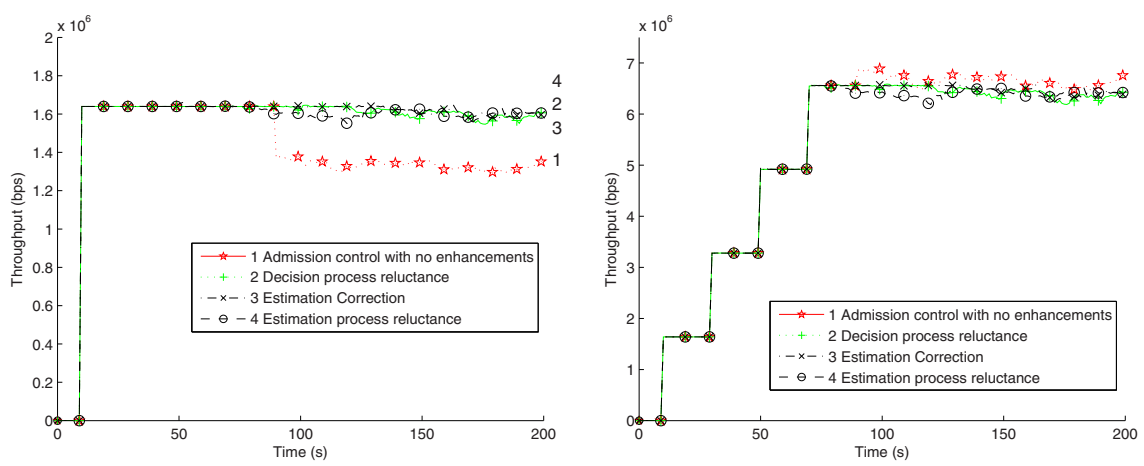

Fig. 4 Mean throughput of flows, scenario 3

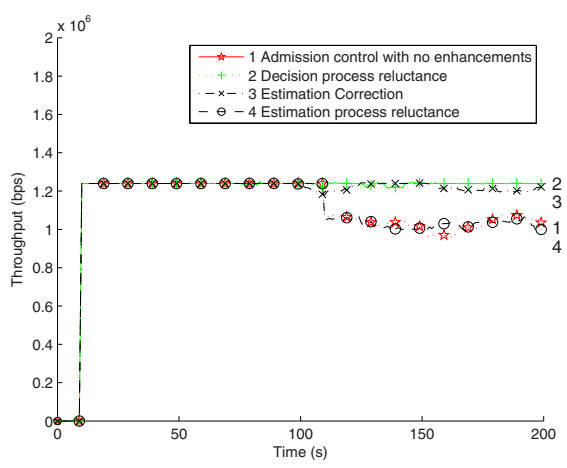

Fig. 5 Total throughput of flows, scenario 3

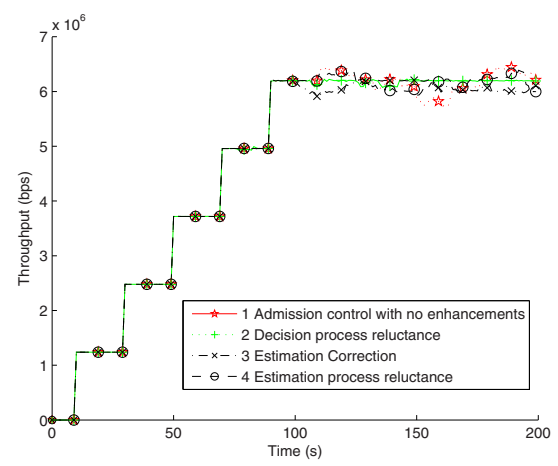

Fig. 6 Mean throughput of flows, scenario 3

Fig. 7 Total throughput of flows, scenario 3
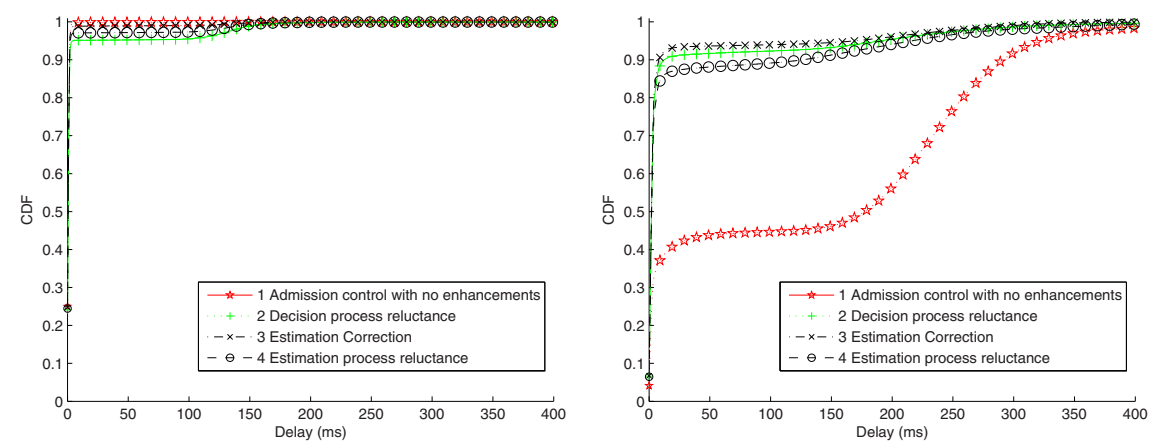

Fig. $8 \mathrm{CDF}$ of delays, scenario 1

Fig. 9 CDF of delays, scenario 2

duction of the proposed modifications. It can be clearly seen in the following that two of the three proposed modifications achieve a correction of the problems of the original algorithm. 


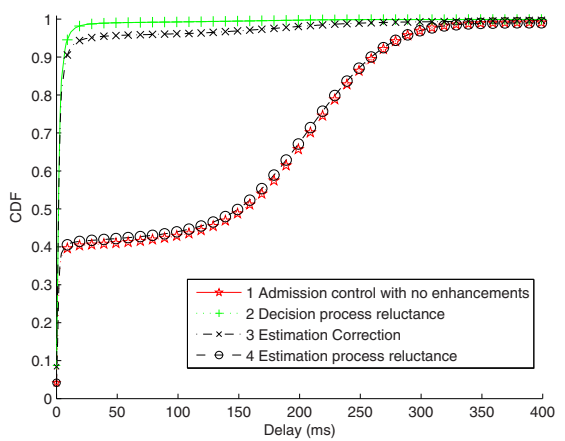

Fig. 10 CDF of delays, scenario 3

\section{Scenariol}

The results are presented in figures $2,3,8$. Scenario 1 is a case where no bad admission decisions were made by the admission control algorithm. It can be seen that the enhancements proposed did not degrade the service offered by the admission control algorithm. The enhancements admitted the ideal number of flows: maximizing the utilization of the medium without degrading the service offered to the active flows (fig. 2-3). As we said earlier, the main aim of the enhancements is to make admission decisions more drastic in order to avoid a bad admission decision. Here, no bad decisions were taken, neither by the original admission control algorithm nor by the enhanced algorithm: the mean throughput per flow respects each flows request and the delays are minimal (fig. 8).

\section{Scenario 2}

The results are presented in figures 4, 5, 9. In scenario 2, the original algorithm will admit one too many flows. The enhancements will correct this flaw. This will result in a better mean throughput per flow (fig. 4) (better in the way it respects the admitted flows requests) and a better distribution of delays (fig. 9) (with the enhancements, depending on the case, $90 \%$ of the packets have delays less than $10 \mathrm{~ms}$ whereas it is the case for only $37 \%$ of the packets in the scenario without enhancements). The flow that was admitted in excess by the original algorithm will cause unexpectedly additional collisions which will in turn cause the service provided to be degraded.

\section{Scenario 3}

The results are presented in figures $6,7,10$. The same analysis can be made here with the difference that the estimation process enhancement presented in section 3.1.1 will not correct the admission control algorithm's flaw in this scenario. It is mostly the case with flows with small bandwidth demand requesting admission: 
when the admission control algorithm makes a bad decision about admitting a flow with a smaller requested bandwidth the estimation process enhancement will not correct it. This enhancement acting mainly in a way linear with the new flow's request, its effect will be lessened.

\section{Conclusion}

We have proposed, in this paper, modifications to an hybrid admission control algorithm for IEEE 802.11e EDCA with the objective of rendering the algorithm more reluctant to admit a flow in a high occupancy rate of the medium: so as to avoid bad admission decisions made by the original algorithm. Simulations were made showing the behavior of each of the modifications in varying scenarios. Two of the proposed modifications corrected the original algorithm's problem. We would plead for the busy probability correction modification to be used as it is simpler to implement, more logical and with a better performance in most cases. Future work include implementing the algorithm presented here along with the enhancements and testing it within an experimental WMM (Wireless Multimedia) platform.

\section{References}

1. International Standard for Telecommunications and Information Exchange between Systems - LAN/MAN specific Requirements - Part 11: Wireless LAN MAC and PHY specifications, 1999

2. IEEE Standard for Telecommunications and Information Exchange between Systems LAN/MAN specific Requirements - Part 11: Wireless LAN MAC and PHY specifications - Amendment 8: Medium Access Control QoS Enhancements, November 2005

3. Mohamad El Masri and Guy Juanole and Slim Abdellatif: Revisiting the Markov chain model of IEEE 802.11e EDCA and introducing the virtual collision phenomenon, proceedings of the International Conference on Wireless Information Networks and Systems, WINSYS '07, July 2007

4. Mohamad El Masri and Guy Juanole and Slim Abdellatif, A synthetic model of IEEE 802.11e EDCA, proceedings of the International Conference on Latest Advances in Networks, ICLAN '07, December 2007

5. Mohamad El Masri and Guy Juanole and Slim Abdellatif, Hybrid admission control algorithm for IEEE 802.11e EDCA: analysis, the International Conference on Networks, ICN '08', April 2008

6. Deyun Gao and Jianfei Cai and King Ngi Ngan, Admission control in IEEE 802.11e wireless LANs, IEEE Network, vol. 19, pp. 6-13, July-August 2005

7. Dennis Pong and Tim Moors, Call admission control for IEEE 802.11 contention access mechanism ,IEEE Global Telecommunications Conference, GLOBECOM '03, December 2003

8. The network Simulator - ns-2, http: / / www. isi.edu/nsnam/ns /

9. An IEEE 802.11e EDCA and CFB Simulation Model for ns-2, http://www.tkn. tu-berlin.de/research/802.11e_ns2/ 